\title{
On the Throughput of Mixed FSO/RF UAV-Enabled Mobile Relaying Systems with a Buffer Constraint
}

\author{
Ju-Hyung Lee ${ }^{\dagger}$, Ki-Hong Park ${ }^{\ddagger}$, Mohamed-Slim Alouini ${ }^{\ddagger}$, and Young-Chai Ko ${ }^{\dagger}$ \\ ${ }^{\dagger}$ Electrical and Computer Engineering, Korea University, Seoul, Korea \\ ${ }^{\ddagger}$ Computer, Electrical and Mathematical Science and Engineering (CEMSE) Division \\ King Abdullah University of Science and Technology (KAUST), Thuwal, Saudi Arabia \\ leejuhyung@korea.ac.kr, \{kihong.park,slim.alouini\}@kaust.edu.sa, koyc@korea.ac.kr
}

\begin{abstract}
In this paper, we consider an unmanned aerial vehicle (UAV) aided mobile relaying system under a buffer constraint at the relay node. We propose a new relaying protocol employing mixed free-space optical/radio frequency (FSO/RF) communication, i.e., the source-relay and relay-destination link utilize FSO communication and RF communication, respectively, under the buffer constraint which is required to consider practical relay system. We study the trajectory optimization problem of buffer-constrained UAV-relaying in order to maximize the end-to-end data throughput. Taking the conditions of the mixed FSO/RF systems (e.g., a full-duplex relaying network that works in decode-and-forward, atmospheric attenuation, transmit power, and bandwidth in FSO and RF links) into consideration, we characterize the channel and throughput models. Furthermore, corresponding to the buffer-aided relaying, we derive a limited buffer constraint regarding the state of the queue in the buffer of relay. We solve the optimal trajectory problem of the UAV to maximize the throughput of user terminal using quadratically constrained programming. As a result, we propose an iterative algorithm that efficiently finds a local optimum solution for the throughput maximization problems. Our numerical results show that proposed buffer-aided mobile relaying achieves $161.3 \%$ throughput gains compared to a static relaying scheme.
\end{abstract}

\section{INTRODUCTION}

As it becomes easier to implement unmanned aerial vehicles (UAVs), UAV-aided mobile relaying systems have attracted great research interest. Motivated by the mounting interest in the unmanned flying platforms, the feasibility of a UAVenabled mobile relaying framework, where it transports the backhaul/fronthaul traffic between the access and core networks, has been investigated [1], [2]. Compared to a conventional static relaying system, the mobile relaying has several key advantages. Above all, cost-effectiveness and easy deployment make the mobile relaying systems especially suitable for unexpected or temporary events, such as emergency response, military operation, etc. In addition, its high mobility offers new opportunities for delay-tolerant applications (e.g., periodic sensing, large data uploading/downloading) and performance improvements through the dynamic relay relocations to achieve the better communication environment [3]. Especially in fifth-generation (5G) and future wireless networks, wireless backhaul/fronthaul networks are required to meet a super high rate requirements, particularly in the presence of ultra-dense heterogeneous small cells. Accordingly, several groups of IT business and academia have focused on this challenge and investigated a free-space optical communication (FSO)-based UAV-enabled relaying as a promising solution [4], [5].

Extensive works have considered the FSO based communication as an attractive solution to explosive rate requirements in communications and have studied mixed RF/FSO system to take advantage of both RF and FSO links. The authors in [6]-[8] investigated the end-to-end performance analysis of the mixed RF/FSO. In [7], the authors provided a generalized framework for performance analysis of mixed RF/FSO systems adopting the most generalized turbulent fading model. Based on the high signal-to-noise-ratio (SNR) analysis, the authors also provided a diversity gain analysis and discussed the bottlenecks in the mixed RF/FSO systems. In [8], multiple mobile users transmit their data to an intermediate decodeand-forward relay node, through $\mathrm{RF}$ links using a virtual multiple-input multiple-output (MIMO) system, and the relay node forwards the multiplexed data of all users through an FSO link. The authors considered the relay node equipped with a buffer in the physical layer, for temporal storage of the user's data until the best channel conditions at the relay-destination link are met. The results of [8] show that pointing error and severe weather turbulence conditions in FSO link become more tolerable with the existence of the relay's buffer, furthermore, buffering in the physical layer provides a significant enhancement to the system performance.

References [8]-[10] focused on the buffer-aided relaying to take advantage of this buffering. The authors in [9] proposed a new protocol exploiting both relays buffering and mobility, to enhance the system throughput and the end-to-end packet delay under bursty arrivals. The work in [10] proposed the design of FSO-based buffer-aided cooperative protocols and turned out that equipping the FSO relays with buffers can enhance the performance at the expense of delays.

By taking the aforementioned advantages of mobility, we study the throughput of a new mixed FSO/RF-based mobile relaying system. Considering the packet delay due to the difference in achievable data rate between FSO and RF links according to relay's position, we further consider a buffer constraint on this system. Specifically, the main contributions of this work are summarized as below:

- We look into the scenario illustrated in Fig. 1, where dualhop mixed FSO/RF communications are conducted on UAV-aided relaying with a buffer constraint. The buffer 


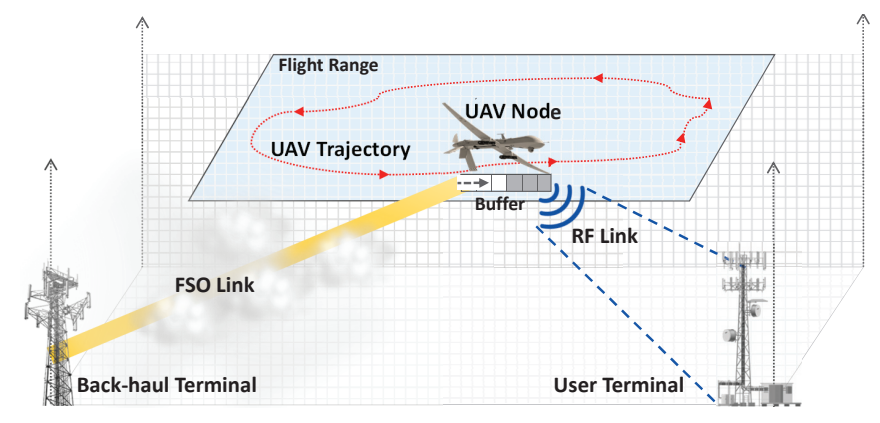

Fig. 1. An illustration of dual-hop mixed FSO/RF communication with help of an UAV-assisted relay.

constraint makes the mobile relay to cycle between the backhaul terminal and the user terminal to prevent a buffer overflow. Since mobile relay need a proper path to satisfy this buffer constraint, we address a trajectory optimization for throughput maximization. To the best of our knowledge, there is no open literature to address the optimization of the buffer-aided mobile relaying system with mixed FSO/RF links.

- To tackle these non-convex problems, we propose an iterative algorithm by adopting the successive optimization method to obtain the local optimal solution. To be specific, we optimize the UAV's trajectory. The trajectory can be determined by applying quadratically constrained programming (QCP)

- Under the different conditions, e.g., visibility, bandwidth difference in each link, and buffer size, the throughput maximized trajectories are presented. We validate the superiority of the proposed compared to the conventional scheme, through the simulation and numerical results.

The remainder of the paper is organized as follows: In Section II, the considered system and channel models are presented. The throughput maximization problem for buffer-aided mobile relaying with a limited buffer is formulated and optimized in Section III. In Section IV, numerical results are presented, and some conclusions are drawn in Section V.

\section{System MOdEL}

We consider a dual-hop mixed FSO/RF communication via on the UAV-assisted relay as illustrated in Fig. 1. Specifically, the UAV-aided relay node employs FSO for receiving information from a backhaul terminal and RF communication for forwarding information to a user terminal ${ }^{1}$. Based on three dimensional Cartesian coordinates for the location of the terminals, we assume that the backhaul terminal and the user terminal located at position $\mathbf{q}_{\mathcal{S}}=[0,0,0]^{T}$ and $\mathbf{q}_{\mathcal{D}}=[L, 0,0]^{T}$, respectively, while the UAV flies at a constant altitude of $H$ with a predetermined maximum speed $V_{\max }$ and acceleration

\footnotetext{
${ }^{1}$ We can also assume the opposite uplink situation, e.g., FSO for forwarding information to the backhaul terminal and RF communication for receiving information from the user terminal(s), such as the application of information collection. Note that the extension of the mixed FSO/RF UAV-enabled mobile relaying design to the more general cooperative system remains to be our future work.
}

$A_{\max }$ for a period $T$. The time-varying coordinate of the UAV node can be denoted as $\mathbf{q}_{\mathcal{R}}(t)=\left[x_{\mathcal{R}}(t), y_{\mathcal{R}}(t), H\right]^{T} \in \mathbb{R}^{3 \times 1}$, $0 \leq t \leq T$.

For ease of analysis, we consider a discrete-time model in this systems as in [5]. The time horizon $T$ is divided into $N$ time intervals each with duration $\delta_{t}$, i.e., $T=N \cdot \delta_{t}$. The duration $\delta_{t}$ is chosen to be sufficiently small so that the UAV's location is to be adequately approximated within each slot. Accordingly, the UAV's location $\mathbf{q}_{\mathcal{R}}(t)$ can be approximated in a discrete-time model, i.e., $\mathbf{q}_{\mathcal{R}}[n] \triangleq \mathbf{q}_{\mathcal{R}}\left(n \delta_{t}\right)=$ $\left[x_{\mathcal{R}}\left(n \delta_{t}\right), y_{\mathcal{R}}\left(n \delta_{t}\right), H\right]^{T}=\left[x_{\mathcal{R}}[n], y_{\mathcal{R}}[n], H\right]^{T} \in \mathbb{R}^{3 \times 1}, 0 \leq$ $n \leq N+1$

Since we consider channel characteristics of air-to-ground links between the UAV and ground terminals and an offline trajectory optimization, we assume line-of-sight (LoS) links without Doppler effect as in [3], [11]. Therefore, the deterministic propagation models are adopted under the position of the UAV and attenuation conditions in this paper. In the following, we present channel and transmission rate models for FSO and RF communication, respectively, and introduce a buffer constraint that describes the queuing system of a practical relay with a finite size of the buffer.

\section{A. System Model for the FSO Link}

In FSO, the channel gain at a link distance $l_{\mathrm{FSO}}$, based on the Beer-Lambert law which relates to the signal attenuation, can be expressed as

$$
h_{\mathrm{FSO}}[n]=e^{-\beta \cdot l_{\mathrm{FSO}}[n]}=e^{-\beta \cdot\left\|\mathbf{q}_{\mathcal{R}}[n]-\mathbf{q}_{\mathcal{S}}\right\|}, n=1, \cdots, N,
$$

where $\beta_{\mathrm{dB}}=\frac{3.91}{V}\left(\frac{\lambda}{550 \mathrm{~nm}}\right)^{-p}[\mathrm{~dB} / \mathrm{km}]$ value depends on the wavelength $\lambda$ assumed to be $1550 \mathrm{~nm}$ in this paper, $V$ is the visibility in $\mathrm{km}$, and the size distribution coefficient $p$ determines by Kim model [12]. Note that $\beta=\frac{\beta_{\mathrm{dB}}}{10^{4} \log _{10} e}\left[\mathrm{~m}^{-1}\right]$.

While the capacity of FSO has not been known in closedform, capacity bounds of FSO have been proposed in several papers. In this paper, we use the lower bound of FSO capacity introduced in [13], for describing data rate of FSO link between source and relay (i.e., backhaul terminal and UAVenabled mobile relay). The average optical SNR (ASNR) is denoted as $\gamma_{\mathrm{FSO}}^{2}=\frac{\varepsilon^{2}}{\sigma_{\mathrm{FSO}}^{2}}$ where $\varepsilon$ and $\sigma_{\mathrm{FSO}}^{2}$ are the average optical power and noise variance for FSO, respectively. The parameter related to $\mathrm{ASNR}, k_{1}$ and parameter related to attenuation condition, $k_{2}$ are formulated as

$$
k_{1}=\left\{\begin{array}{ll}
\frac{e^{2 \alpha \mu^{*}}}{2 \pi e}\left(\frac{1-e^{-\mu^{*}}}{\mu^{*}}\right)^{2} \frac{\gamma^{2}}{\alpha^{2}} & \text { if } 0<\alpha<\frac{1}{2} \\
\frac{\gamma^{2}}{2 \pi e \alpha^{2}} & \text { if } \frac{1}{2}<\alpha<1
\end{array}, \quad k_{2}=2 \beta .\right.
$$

Note that $\mu$ is the free parameter which indicates the solution to the equation $\alpha=\frac{1}{\mu^{*}}-\frac{e^{-\mu^{*}}}{\left(1-e^{-\mu^{*}}\right)}$ when the average-to-peak ratio (APR) is set to $\alpha=\frac{\varepsilon}{\Lambda}$ where $\Lambda$ is peak optical power.

The transmission rate of FSO in bits/second (bps) for the slot $n$ can be expressed with the channel gain for FSO link (1), the parameters in (2), the bandwidth of FSO $B_{\mathrm{FSO}}$, and the received ASNR $\bar{\gamma}_{\mathrm{FSO}}^{2}=h_{\mathrm{FSO}}^{2} \cdot \gamma_{\mathrm{FSO}}^{2}$ as follows: 


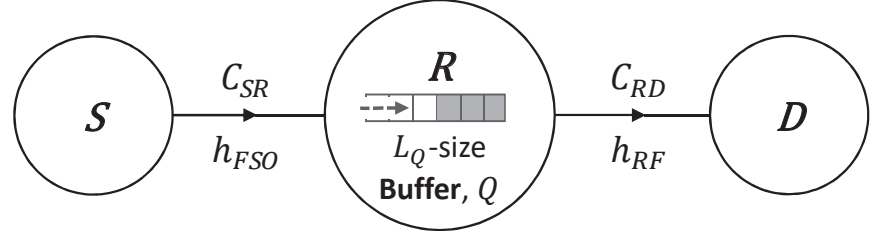

Fig. 2. System model for a buffer-aided relay network with a mixed FSO/RF communication.

$$
\begin{array}{r}
R_{\mathrm{FSO}}[n]=\frac{B_{\mathrm{FSO}}}{2 \log 2} \cdot \log \left(1+k_{1} e^{-k_{2} \cdot\left\|\mathbf{q}_{\mathcal{R}}[n]-\mathbf{q}_{\mathcal{S}}\right\|}\right)[\mathrm{bps}], \\
n=1, \cdots, N .
\end{array}
$$

\section{B. System Model for the RF Link}

The channel gain of RF link $h_{\mathrm{RF}}$ between UAV and user terminal at a link distance $l_{\mathrm{RF}}$ can be expressed as

$$
h_{\mathrm{RF}}[n]=\sqrt{\frac{\beta_{0}}{l_{\mathrm{RF}}^{2}[n]}}=\sqrt{\frac{\beta_{0}}{\left\|\mathbf{q}_{\mathcal{D}}-\mathbf{q}_{\mathcal{R}}[n]\right\|^{2}}}, \quad n=1, \cdots, N,
$$

where $\beta_{0}$ represents the received power at the reference distance $d_{0}=1[\mathrm{~m}]$. Accordingly, the transmission rate of $\mathrm{RF}$ in [bps] for the slot $n$ can be expressed as

$$
\begin{array}{r}
R_{\mathrm{RF}}[n]=B_{\mathrm{RF}} \cdot \log _{2}\left(1+\frac{\gamma_{0}}{\left\|\mathbf{q}_{\mathcal{D}}-\mathbf{q}_{\mathcal{R}}[n]\right\|^{2}}\right)[\mathrm{bps}], \\
n=1, \cdots, N,
\end{array}
$$

where $B_{\mathrm{RF}}$ represents the RF bandwidth, and $\gamma_{0}=\frac{\beta_{0} \cdot P}{\sigma_{\mathrm{RF}}^{2}}$ indicates reference SNR with the constant transmission power $P$ and the noise variance $\sigma_{R F}^{2}$.

\section{Buffer Constraint at UAV-assisted Relay}

Consider a dual-hop mixed FSO/RF network communicating between a source link $\mathcal{S}$ and a destination $\mathcal{D}$ via a single UAV-enabled mobile relay node denoted by $\mathcal{R}$, as shown in Fig. 2. Throughout the system, we assume that the source transmits with FSO at the rate of $R_{\mathrm{FSO}}[n]$ and the data is organized in packets. For ease of analysis, we consider the amount of data rate instead of the packet rate. We consider first-in-first-out (FIFO) for the scheduling policy at queuing node, which states that packets are enqueued in turn and the packets that wait longest in a buffer is dequeued first. Moreover, we leverage a full-duplex relaying (FDR) which works in decode-and-forward (DF) protocol in the system, by taking account of the mixed FSO/RF characteristic that it has no self-interference [10]. In the following, we discuss on the queuing dynamics when source and relay transmit data as in [14].

Source transmits: As FSO is chosen for $\mathcal{S}-\mathcal{R}$ link, the number of bits transmitted by the source in time slot $n$ is given by

$$
C_{\mathcal{S R}}[n]=R_{\mathrm{FSO}}[n], n=1, \cdots, N \text {. }
$$

Accordingly, the number of bits enqueued in the buffer but not yet dequeued, i.e., remaining bits in the buffer of relay can be expressed as

$$
\begin{array}{r}
Q[n]=\min \left\{Q[n-1]+C_{\mathcal{S R}}[n]-C_{\mathcal{R} \mathcal{D}}[n], L_{\mathrm{Q}}\right\}, \\
n=2, \cdots, N,
\end{array}
$$

where $L_{\mathrm{Q}}$ indicates buffer size, $Q[1]=R_{\mathrm{FSO}}[1], Q[0]=0$, $C_{\mathcal{R D}}[1]=0$. Note that $C_{\mathcal{R D}}[n]$ denotes the bits received by the destination (i.e., the user terminal) in time slot $n$, which can be expressed as below:

\section{Relay transmits:}

$$
\begin{array}{r}
C_{\mathcal{R D}}[n]=\min \left\{R_{\mathrm{RF}}[n], Q[n-1]+C_{\mathcal{S R}}[n]\right\}, \\
n=2, \cdots, N,
\end{array}
$$

where we consider that the maximal number of transmittable bits at the relay is limited by the remaining bits in the buffer or the instantaneous capacity for $\mathcal{R}-\mathcal{D}$ link which uses the RF link.

Thus, we can formulate the average throughput in the mixed FSO/RF communication with the limited buffer as follows:

$$
\begin{aligned}
\Phi & =\frac{1}{N-1} \sum_{n=2}^{N} C_{\mathcal{R \mathcal { D }}}[n] \\
& =\mathbb{E}\left[\min \left\{Q[n-1]+C_{\mathcal{S R}}[n], R_{\mathrm{RF}}[n]\right\}\right] .
\end{aligned}
$$

The goal of the following section is the maximization of $\Phi$ by the optimization of UAV's trajectory.

\section{Throughrut MAXimization With A Limited BUFFER}

In this section, we tackle the trajectory optimization of UAVaided relay to maximize the throughput of mixed FSO/RF under UAV mobility and buffer constraints. The following problem, P0, corresponds to the average throughput maximization, under the constraint related to an actual flight condition of UAV, without a buffer constraint.

$$
\begin{aligned}
(\mathrm{P} 0) \max & \Phi \\
\text { s.t } \quad & \mathbf{v}_{\mathcal{R}}[n+1]=\mathbf{v}_{\mathcal{R}}[n]+\mathbf{a}_{\mathcal{R}}[n] \delta_{t} \\
& \mathbf{q}_{\mathcal{R}}[n+1]=\mathbf{q}_{\mathcal{R}}[n]+\mathbf{v}_{\mathcal{R}}[n] \delta_{t}+\frac{1}{2} \mathbf{a}_{\mathcal{R}}[n] \delta_{t}^{2} \\
& n=0, \cdots, N \\
& \mathbf{v}_{\mathcal{R}}[0]=\mathbf{v}_{\mathrm{I}}, \mathbf{v}_{\mathcal{R}}[N+1]=\mathbf{v}_{\mathrm{F}} \\
& \left\|\mathbf{a}_{\mathcal{R}}[n]\right\| \leq A_{\max }, n=0, \cdots, N \\
& \left\|\mathbf{v}_{\mathcal{R}}[n]\right\| \leq V_{\max }, n=1, \cdots, N
\end{aligned}
$$

Note that the equality constraint (10) characterizes the discrete state-space model of UAV's location and velocity related to the position $\mathbf{q}_{\mathcal{R}}[n]$, the velocity $\mathbf{v}_{\mathcal{R}}[n]$, as well as the acceleration $\mathbf{a}_{\mathcal{R}}[n]$. To take UAV's movement into account, UAV is constrained with the initial and final velocities in (11), the maximum acceleration in (12), and maximum velocity in (13). 
In addition to UAV's flight constraints, we also consider the buffer constraint of relay and the mobile relay constraints. With the information-causality constraint as in [15], i.e., the condition that relay can only transmit the data that has been previously received from the source, the objective $\Phi$ can be rewritten $^{2}$ as $\sum_{n=2}^{N} R_{\mathrm{RF}}[n]$. Moreover, we can establish the buffer constraint derived with (7). Thus, we can rewrite the following (P1) as (P0) considering the buffer constraints with the $L_{\mathrm{Q}}$-size buffer and the mobile relay-related constraints.

$$
\begin{array}{cl}
\max _{\substack{\mathbf{q}_{\mathcal{R}}[n], \mathbf{v}_{\mathcal{R}}[n] \\
\mathbf{a}_{\mathcal{R}}[n] \\
\text { s.t }}} & \sum_{n=2}^{N} R_{\mathrm{RF}}[n] \\
& (10)-(13), \\
& Q[n] \leq L_{\mathrm{Q}}, \\
& \sum_{i=2}^{n} R_{\mathrm{RF}}[i] \leq \sum_{i=1}^{n-1} R_{\mathrm{FSO}}[i], \\
& n=2, \cdots, N, \\
& \left\|\mathbf{q}[n]-\mathbf{q}_{\mathrm{b}}\right\| \leq r_{\mathrm{b}}, \forall n, \\
& \|\mathbf{q}[N]-\mathbf{q}[1]\| \leq \delta_{t} V_{\max },
\end{array}
$$

where (14) represents the buffer constraint that prevents the current queue size in the buffer $Q[n]$ to overflow the limited buffer size $L_{\mathrm{Q}}$, the constraint (16) describes the boundary constraint which makes mobile relay fly inside a circle with the radius $r_{\mathrm{b}}$ around the center $\mathrm{q}_{\mathrm{b}}$, and (17) expresses the recurrence constraint as in [16] that it forces the mobile relay to fly back to the initial position.

Despite of the convex constraints (10)-(13), (16), and (17), the non-concave objective function $R_{\mathrm{RF}}[n]$ and non-convex constraints (14)-(15) cause (P1) to be non-convex optimization problem, which therefore can not be addressed with standard convex optimization method.

To tackle such the non-convex problem, firstly, we use the first-order Taylor approximation to $R_{\mathrm{RF}}[n]$. Accordingly, with any given local value $\mathbf{q}_{\mathcal{R}}^{k}[n]$ at the iteration $k$, we have approximated throughput of $\mathrm{RF}$ as $R_{\mathrm{RF}}^{\prime}[n]=$ $B_{\mathrm{RF}} \quad \cdot \quad\left(\mathcal{A}^{k}-\mathcal{B}^{k}\left(\left\|\mathbf{q}_{\mathcal{D}}-\mathbf{q}_{\mathcal{R}}[n]\right\|^{2}-\left\|\mathbf{q}_{\mathcal{D}}-\mathbf{q}_{\mathcal{R}}^{k}[n]\right\|^{2}\right)\right)$ where $\mathcal{A}^{k}=\log _{2}\left(1+\frac{\gamma_{0}}{\left\|\mathbf{q}_{\mathcal{D}}-\mathbf{q}_{\mathcal{R}}^{k}[n]\right\|^{2}}\right), \quad \mathcal{B}^{k}=$ $\frac{\gamma_{0} \cdot \log _{2} e}{\left(\gamma_{0}+\left\|\mathbf{q}_{\mathcal{D}}-\mathbf{q}_{\mathcal{R}}^{k}[n]\right\|^{2}\right)\left(\left\|\mathbf{q}_{\mathcal{D}}-\mathbf{q}_{\mathcal{R}}^{k}[n]\right\|^{2}\right)}, \quad n=2, \cdots, N$ [11]. Secondly, using high-SNR approximation ${ }^{3}$, we express the lower bounded throughput of FSO, $R_{\mathrm{FSO}}^{\prime}[n]=\frac{B_{\mathrm{FSO}}}{2 \log 2}\left(\log \left(k_{1}\right)-k_{2} \cdot\left\|\mathbf{q}_{\mathcal{R}}[n]-\mathbf{q}_{\mathcal{S}}\right\|\right)$, $n=1, \cdots, N-1$ [17]. Notice that $R_{\mathrm{RF}}^{\prime}[n]$ and $R_{\mathrm{FSO}}^{\prime}[n]$ are concave functions with respect to $\mathbf{q}_{\mathcal{R}}[n]$.

Now, let us replace the non-convex constraint (15) to convex constraint with $\left\{R_{\mathrm{RF}}^{\prime}[n]\right\}_{n=2}^{N},\left\{R_{\mathrm{FSO}}^{\prime}[n]\right\}_{n=1}^{N-1}$, and introduce a slack variable $\{r[n]\}_{n=2}^{N}$ for the non-concave objective

\footnotetext{
${ }^{2}$ The average throughput for the system $\Phi$ in (9) can be dealt equivalently with a total amount of throughput received by the destination, i.e., $\sum_{n=2}^{N} C_{\mathcal{R} \mathcal{D}}[n]$

${ }^{3}$ Although UAV flies on heavy-fog condition which is the worst case, we assume that the part of $R_{\mathrm{FSO}}[n]$ satisfies $k_{1} \cdot e^{-k_{2} \cdot\left\|\mathbf{q}_{\mathcal{R}}[n]-\mathbf{q}_{\mathcal{S}}\right\|} \gg 1$ within the flight range.
}

function. Thus, we can formulate (P1) to the following optimization problem for any given local value $\left\{\mathbf{q}_{\mathcal{R}}^{k}[n]\right\}_{n=1}^{N}$ at the $k$-th iteration.

$$
\begin{aligned}
\left(\mathrm{P}^{\star}\right) \max _{\begin{array}{c}
r[n], d_{\mathrm{sr}}[n], \\
\mathbf{q}_{\mathcal{R}}[n], \mathbf{v} \mathcal{R}[n], \\
\mathbf{a}_{\mathcal{R}}[n] \\
\text { s.t }
\end{array}} & \sum_{n=2}^{N} r[n] \\
& (10)-(13),(16)-(17), \\
& \sum_{i=1}^{n} R_{\mathrm{FSO}}^{\prime \prime}[i]-\sum_{i=2}^{n} r[i] \leq L_{\mathrm{Q}} \\
& n=2, \cdots, N, \\
& d_{\mathrm{sr}}^{2}[n] \leq \Gamma\left(\mathbf{q}_{\mathcal{R}}[n]\right), n=1, \cdots, N, \\
& \sum_{i=2}^{n} r[i] \leq \sum_{i=1}^{n-1} R_{\mathrm{FSO}}^{\prime}[i], \\
& r[n] \leq R_{\mathrm{RF}}^{\prime}[n] \\
& n=2, \cdots, N,
\end{aligned}
$$

where we can derive $\Gamma\left(\mathbf{q}_{\mathcal{R}}[n]\right)=\left\|\mathbf{q}_{\mathcal{R}}^{k}[n]-\mathbf{q}_{\mathcal{S}}\right\|^{2}+2\left(\mathbf{q}_{\mathcal{R}}^{k}[n]-\right.$ $\left.\mathbf{q}_{\mathcal{S}}\right)^{T} \cdot\left(\mathbf{q}_{\mathcal{R}}[n]-\mathbf{q}_{\mathcal{R}}^{k}[n]\right), n=1, \cdots, N$ as the first Taylor approximation of $\left\|\mathbf{q}_{\mathcal{R}}[n]-\mathbf{q}_{\mathcal{S}}\right\|^{2}$ at the local point $\mathbf{q}_{\mathcal{R}}^{k}[n]$ obtained at the $k$-th iteration. Note that $R_{\mathrm{FSO}}^{\prime \prime}[n]=$ $\frac{B_{\mathrm{FSO}}}{2 \log 2}\left(\log \left(k_{1}\right)-k_{2} \cdot d_{\mathrm{sr}}[n]\right), n=1, \cdots, N$ in which the upper bound of $\Gamma\left(\mathbf{q}_{\mathcal{R}}[n]\right),\left\{d_{\mathrm{sr}}^{2}[n]\right\}_{n=1}^{N}$, is used for the nonconvex constraint (14). The slack variable $r[n]$ is introduced for dealing with the non-concave objective $R_{\mathrm{RF}}[n]$. Thus, we establish (18)-(19) with the auxiliary variable $d_{\mathrm{sr}}[n]$ for (14) and set (20)-(21) to address the non-convex constraints (15). Note that constraint (18) can be derived with (14) under the information-causality constraint (15).

The type of optimization problem $\left(\mathrm{P} 1^{\star}\right)$ is the convex quadratically constrained program (QCP). The convex QCP can be solved within a polynomial complexity, by standard convex optimization solvers such as CVX. Then, we can suboptimally solve (P1) via the successive convex optimization to $\left(\mathrm{P} 1^{\star}\right)$ iteratively updating the local point $\left\{\mathbf{q}_{\mathcal{R}}^{k}[n]\right\}_{n=1}^{N}$. Note that it has been proved that the successive convex optimization method converges to at least a local optimal point [15].

In closing the section, we summarize the proposed successive process for (P1) in Algorithm 1.

Note that depending on an application, we can also apply Algorithm 1 to (P0), i.e., the case of average throughput maximization without the buffer constraint (14)-(15) and the constraint related to mobile relay (16)-(17).

\section{Numerical RESUlt}

In this section, we provide some selected numerical results to validate our proposed mixed FSO/RF UAV-enabled mobile relaying system with a buffer. We consider a system with the altitude of UAV $H=100 \mathrm{~m}$, the location of backhaul terminal $\mathbf{q}_{\mathcal{S}}=[0,0,0]^{T}$, the location of user terminal $\mathbf{q}_{\mathcal{D}}=[L, 0,0]^{T}$ where $L=2000 \mathrm{~m}$. For the UAV-enabled mobile relaying system, we assume that initial/final velocity 

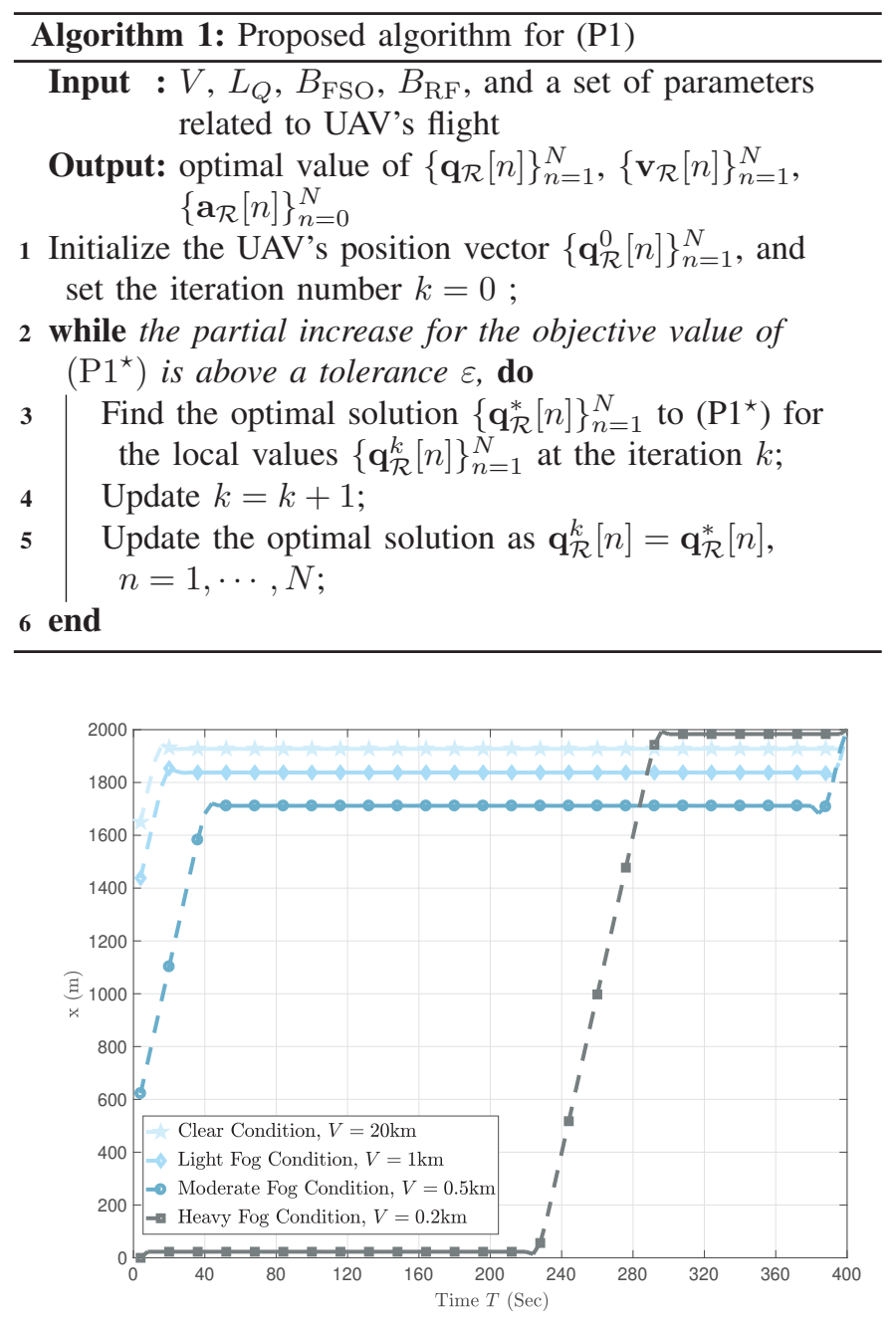

Fig. 3. Throughput maximized UAV's X-coordinate position over time $T$ with respect to the visibility $V$.

$\mathbf{v}_{\mathrm{I}}=\mathbf{v}_{\mathrm{F}}=30\left(\mathbf{q}_{\mathcal{D}}-\mathbf{q}_{\mathcal{S}}\right) /\left\|\mathbf{q}_{\mathcal{D}}-\mathbf{q}_{\mathcal{S}}\right\|$, the maximum velocity $V_{\max }=50 \mathrm{~m} / \mathrm{s}$, and the maximum acceleration $A_{\max }=5$ $\mathrm{m} / \mathrm{s}^{2}$. Unless stated otherwise, we set the time-step size $\delta_{t}=1$ $\mathrm{s}$, the visibility $V=0.2 \mathrm{~km}$, the buffer size $L_{\mathrm{Q}}=10^{9}$, the bandwidth for FSO and RF $B_{\mathrm{FSO}}=B_{\mathrm{RF}}=1 \mathrm{MHz}$, the ASNR $\gamma_{\mathrm{FSO}}=30 \mathrm{~dB}\left(\alpha=\frac{1}{4}\right)$, and the reference SNR $\gamma_{0}=70 \mathrm{~dB}$. The simulation results of this paper are obtained through CVX.

Fig. 3 represents the throughput maximized path with the infinite buffer constraint (i.e. $L_{\mathrm{Q}}=\infty$ ), and without the flight range constraint (16) and the recurrence constraint (17), i.e., the throughput maximized path for (P0). This figure represents the optimized trajectories in $\mathrm{X}$-axis over different atmospheric conditions, under the UAV's flight constraints. In Fig. 3, the better the atmospheric condition (i.e., the better the communication environment of the FSO link), the shorter the mobile relay flies near the backhaul terminal, and then the faster it gets to the user terminal for forwarding data accumulated in the buffer.

Analyzing the buffer constrained throughput maximization (P1), Figs. 4, 5, 6, and 7 are plotted with the boundary con-

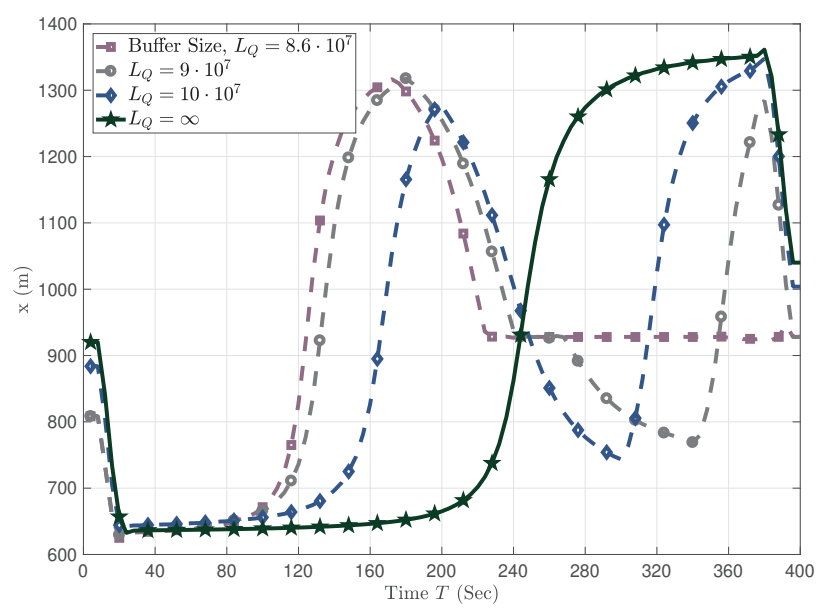

Fig. 4. Throughput maximized UAV's X-coordinate position over time $T$ with respect to the buffer size $L_{\mathrm{Q}}$.

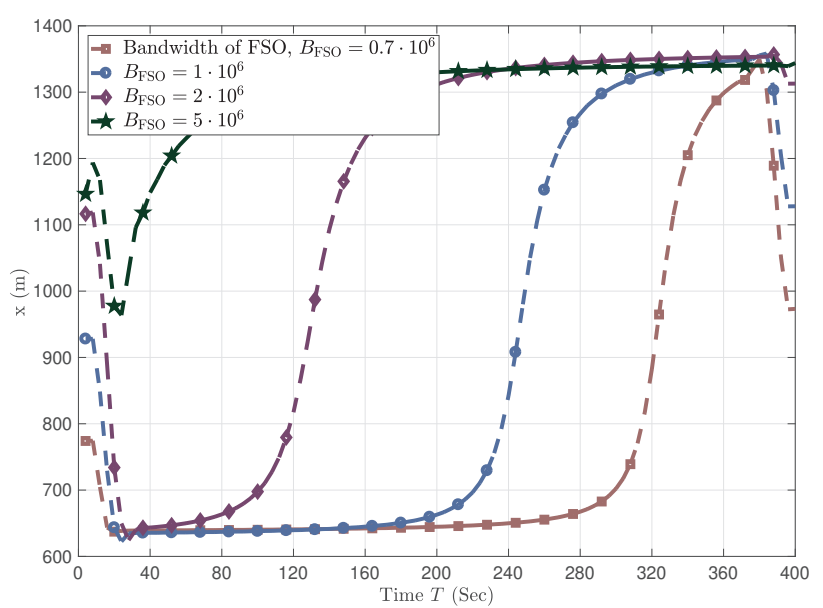

Fig. 5. Throughput maximized UAV's X-coordinate position over time $T$ with respect to the bandwidth of FSO $B_{\mathrm{FSO}}$.

straint where it draws a circle with the origin $[L / 2,500, H]^{T}$ and the radius of the boundary $r_{b}=500 \mathrm{~m}^{4}$.

Fig. 4 shows the throughput maximized trajectory by taking account of the finite buffer size. Furthermore, Figs. 5 and 6 show the throughput optimized trajectory considering the bandwidth difference between the FSO link and RF link. Depending on the different buffer sizes and the different bandwidth size of the FSO link, the optimized trajectories in $\mathrm{X}$-coordinate for the mobile relay are plotted in Fig. 4 and Figs. 5 and 6, respectively.

Fig. 4 gives the insight that a limited buffer size forces the UAV to come and go between the backhaul terminal and the user terminal. The smaller the buffer size, the more frequent the mobile relay circulates. It is an intuitive situation that can occur when a size of buffer in the mobile relaying system is finite. Besides, we can see that when the buffer size is large, the mobile relay can receive and transmit enough data near

${ }^{4}$ For the practical implementation of the mobile relaying system, the boundary constraint is considered. Note that one can omit or modify this flight range constraint in need. 


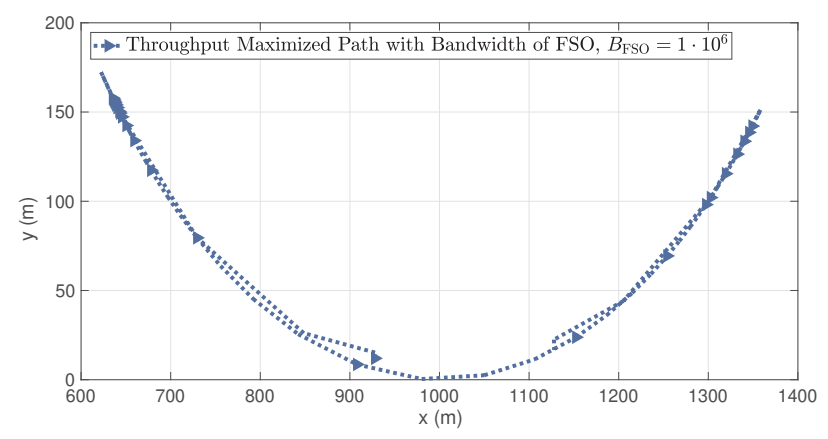

Fig. 6. Throughput maximized UAV's XY-coordinate position over time $T$ with respect to the bandwidth of FSO $B_{\mathrm{FSO}}$.

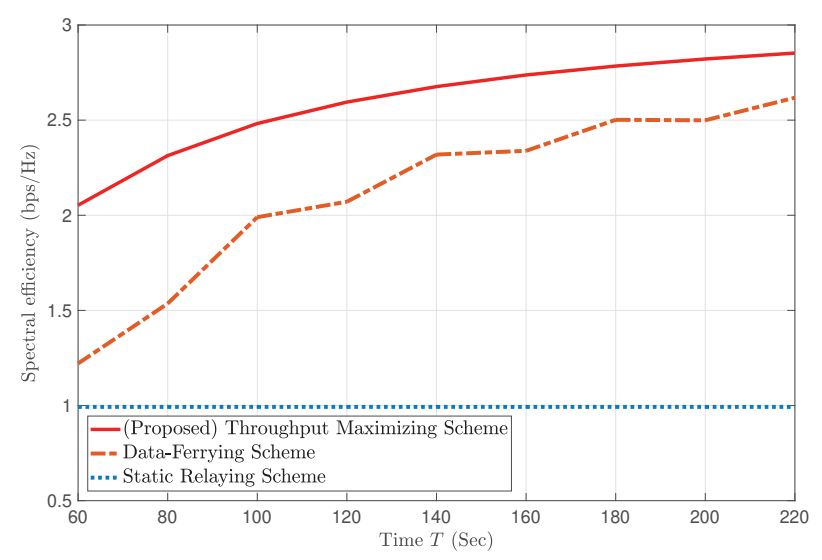

Fig. 7. The comparison study of the proposed work over the conventional works.

the $\mathcal{S}$ link, thus improving the throughput result.

Moreover in Figs. 5 and 6, as the bandwidth of the FSO link increases (i.e., as the achievable rate of the FSO link improves), the mobile relay moves inside the boundary from near the backhaul terminal to near the user terminal more quickly. These trajectories can be intuitively understood as the mobile relay needs to forward the data from $\mathcal{S}$ to $\mathcal{D}$ before the buffer overflow occurs.

As a final remark, Fig. 7 shows the comparison result based on the throughput. This figure shows the superiority of the proposed scheme compared to the baseline schemes (static relaying scheme and data-ferrying scheme where $d_{1}=$ $d_{2}=100 \mathrm{~m}$ [15], [18]). Note that the throughput performance of the proposed throughput maximized path is $161.3 \%$ and $25.29 \%$ larger than those of the static relaying scheme and data-ferrying scheme, respectively.

\section{CONCLUSION}

This paper has investigated the problem of the throughput maximization in mixed FSO/RF UAV-enabled mobile relaying system with a buffer constraint. For the throughput maximization, we have optimized the trajectory of a UAV-assisted relay under the attenuation condition for FSO and the bandwidth difference in mixed FSO/RF that can cause the achievable rate difference. Furthermore, we have practically considered the finite sized buffer, then ascertained the effect of buffer size to the trajectory for UAV-enabled mobile relay. To tackle these non-convex problems, we adopted the successive optimization algorithm. Thus, the trajectory can be determined by applying the proposed algorithm. Through the simulation results, we validated the superiority of the proposed algorithm over the conventional schemes.

\section{ACKNOWLEDGEMENT}

This work was supported by the National Research Foundation of Korea (NRF) grant funded by the Korea government(MSIT)(NRF-2019R1A2B2007789)

\section{REFERENCES}

[1] A. A. Khuwaja, Y. Chen, N. Zhao, M.-S. Alouini, and P. Dobbins, "A survey of channel modeling for UAV communications," IEEE Commun. Surveys Tuts., pp. 1-1, Jul 2018.

[2] Y. Zeng, R. Zhang, and T. J. Lim, "Wireless communications with unmanned aerial vehicles: Opportunities and challenges," IEEE Commun. Mag., vol. 54, no. 5, pp. 36-42, Jul. 2018.

[3] S. Jeong, O. Simeone, and J. Kang, "Mobile edge computing via a UAVmounted cloudlet: Optimization of bit allocation and path planning," IEEE Trans. Veh. Technol., vol. 67, no. 3, pp. 2049-2063, Mar. 2018.

[4] M. Alzenad, M. Z. Shakir, H. Yanikomeroglu, and M.-S. Alouini, "FSO-based vertical backhaul/fronthaul framework for 5G+ wireless networks," IEEE Commun. Mag., vol. 56, no. 1, pp. 218-224, Jan. 2018

[5] P. Zhan, K. Yu, and A. L. Swindlehurst, "Wireless relay communications with unmanned aerial vehicles: Performance and optimization," IEEE Trans. Aerosp. Electron. Syst., vol. 47, no. 3, pp. 2068-2085, Jul. 2011.

[6] H. Samimi and M. Uysal, "End-to-end performance of mixed RF/FSO transmission systems," J. Opt. Commun. Netw., vol. 5, no. 11, pp. 11391144, Nov. 2013.

[7] E. S.-Nasab and M. Uysal, "Generalized performance analysis of mixed RF/FSO cooperative systems," IEEE Trans. Wireless Commun., vol. 15, no. 1, pp. 714-727, Jan. 2016.

[8] Y. F. A.-Eryani, A. M. Salhab, S. A. Zummo, and M.-S. Alouini, "Protocol design and performance analysis of multiuser mixed RF and hybrid FSO/RF relaying with buffers," J. Opt. Commun. Netw., vol. 5, no. 11, pp. 1139-1144, Nov. 2013.

[9] R. Wang, V. K. N. Lau, and H. Huang, "Opportunistic buffered decodewait-and-forward (OBDWF) protocol for mobile wireless relay networks," IEEE Trans. Wireless Commun., vol. 10, no. 4, pp. 1224-1231, Apr. 2011.

[10] C. A.-Rjeily and W. Fawaz, "Buffer-aided relaying protocols for cooperative FSO communications," IEEE Trans. Wireless Commun., vol. 16 no. 12, pp. 8205-8219, Dec. 2017.

[11] Y. Zeng and R. Zhang, "Energy-efficient UAV communication with trajectory optimization," IEEE Trans. Wireless Commun., vol. 16, no. 6, pp. 3747-3760, Jun. 2017.

[12] M. A. Esmail, H. Fathallah, and M.-S. Alouini, "Outdoor FSO communications under fog: Attenuation modeling and performance evaluation," IEEE Photon. J., vol. 8, no. 4, p. 7905622, Aug. 2016.

[13] A. Lapidoth, S. M. Moser, and M. A. Wigger, "On the capacity of free-space optical intensity channels," IEEE Trans. Inf. Theory., vol. 55 no. 10, pp. 4449-4461, Oct. 2009.

[14] N. Zlatanov, R. Schober, and P. Popovski, "Buffer-aided relaying with adaptive link selection," IEEE J. Sel. Areas Commun., vol. 31, no. 8 , pp. 1530-1541, Aug. 2013.

[15] Y. Zeng, R. Zhang, and T. J. Lim, "Throughput maximization for UAV-enabled mobile relaying systems," IEEE Trans. Commun., vol. 64, no. 12, pp. 4983-4996, Dec. 2016.

[16] J. Park, H. Lee, S. Eom, and I. Lee, "Minimum throughput maximization in UAV-aided wireless powered communication networks," arXiv:1801.02781v1 [cs.IT], pp. 1-22, Jan. 2018.

[17] J.-H. Lee, K.-H. Park, M.-S. Alouini, and Y.-C. Ko, "Trajectory optimization of energy efficient FSOC-UAV with atmospheric and geometric loss," in Proc. International Conf. on Ubiquitous and Future Netw., Prague, Czech Republic, 2018, pp. 35-37.

[18] W. Zhao, M. Ammar, and E. Zegura, "A message ferrying approach for data delivery in sparse mobile ad hoc networks," in Proc. ACM MobiHoc., Tokyo, Japan, 2004, pp. 187-198. 